\title{
Long-Range Interactions and Entanglement of Slow Single-Photon Pulses
}

\author{
Inbal Friedler, ${ }^{1}$ David Petrosyan, ${ }^{2}$ Michael Fleischhauer, ${ }^{3}$ and Gershon Kurizki ${ }^{1}$ \\ ${ }^{1}$ Department of Chemical Physics, Weizmann Institute of Science, Rehovot 76100, Israel \\ ${ }^{2}$ Institute of Electronic Structure 85 Laser, FORTH, GR-71110 Heraklion, Crete, Greece \\ ${ }^{3}$ Fachbereich Physik, Universität Kaiserslautern, D-67663 Kaiserslautern, Germany
}

(Dated: November 5, 2018)

\begin{abstract}
We show that very large nonlocal nonlinear interactions between pairs of colliding slow-light pulses can be realized in atomic vapors in the regime of electromagnetically induced transparency. These nonlinearities are mediated by strong, long-range dipole-dipole interactions between Rydberg states of the multi-level atoms in a ladder configuration. In contrast to previously studied schemes, this mechanism can yield a homogeneous conditional phase shift of $\pi$ even for weakly focused singlephoton pulses, thereby allowing a deterministic realization of the photonic phase gate.

PACS numbers: 42.50.Gy, 03.67.Lx
\end{abstract}

Whether or not quantum information processing and quantum computing 1] become practical technologies crucially depends on the ability to implement highfidelity quantum logic gates in a scalable way [2]. Among alternative routes to this challenging goal, are of particular interest the schemes operating with photons as qubits 3, 4], since photons are ideal carriers of quantum information in terms of transfer rates, distances and scalability. A current trend makes use of linear optical elements and photodetectors for the implementation of key components of quantum communications and information processing in a probabilistic way [4]. The desirable objective though is a deterministic realization of entangling operations between individual photons, which require sufficiently strong nonlinearities or long interaction times. These are achievable, at the single-photon level, by tight spatial confinement of the photons, in the very demanding regime of strong atom-field coupling in high- $Q$ cavities [5] .

A promising alternative is to enhance both the nonlinear susceptibility and interaction time, by employing the ultra-slow light propagation in resonant media subject to electromagnetically induced transparency (EIT) [6, 7, 8] . In a pioneering work, Schmidt and Imamoğlu have suggested the possibility of enhanced, non-absorptive, crossphase modulation of two weak fields in the EIT regime [9], provided their interaction time is long enough. However, upon entering the EIT medium light pulses become spatially compressed by the ratio of group velocity $v$ to the vacuum speed of light $c[10$, so that the interaction time of two colliding pulses is a constant independent of $v$. In order to maximize this time, copropagating pulses with nearly matched group velocities have been proposed 11, 12]. The essential drawback of such an approach is the spatial inhomogeneity of the conditional phase shift, causing spectral broadening of the interacting pulses, thereby preventing the realization of a highfidelity quantum phase gate. Alternative approaches free of spectral broadening have been suggested [13, 14, 15]. In all of them, however, a rather tight transverse con- finement through waveguiding or focusing of the pulses, close to the diffraction limit of $\lambda^{2}$, is needed in order to attain a phase shift of $\pi$, which is technically challenging.

When the light pulses enter EIT media, photonic excitations are temporarily transferred to atomic excitations through the formation of quasi-particles, the socalled dark-state (or slow-light) polaritons, which are superpositions of light and matter degrees of freedom [16]. The spatial compression of the pulses leads to an amplification of the matter components of polaritons. In this Letter we propose a hitherto unexplored mechanism for the collisional entanglement of two single-quantum polaritons mediated by the long-range interaction of their matter (atomic) components and demonstrate its effectiveness. In contrast to the previous schemes which employ local interactions, namely either two photons interact with the same atom 11, 12, 13, 14] or two atoms after absorbing the photons undergo $s$-wave scattering [15], here the two polaritons interact via the long-range dipole-dipole interactions between their atomic components in the highly excited Rydberg states. In a static electric field, these internal Rydberg states, populated only in the presence of polaritons, possess large permanent dipole moments [17, which can further enhance the effective interaction time between the polaritons. We will show that under experimentally realizable conditions, the conditional phase shift accumulated during a collision of two single-quantum polaritons is spatially homogeneous and can be sufficiently large for the implementation of the quantum phase gate, even for moderate focusing or transverse confinement of interacting pulses. We note that quantum gates for individual Rydberg atoms, coupled by dipole-dipole interaction, has been proposed in [18].

We consider an ensemble of cold alkali atoms with level configuration as in Fig. 1. All the atoms are initially prepared in the ground state $|g\rangle$. Two weak (quantum) fields $E_{1,2}$ having orthogonal polarizations and propagating in the opposite directions along the $z$ axis resonantly interact with the atoms on the transitions $|g\rangle \rightarrow\left|e_{1,2}\right\rangle$, 
(a)

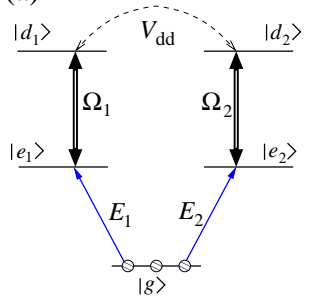

(b)

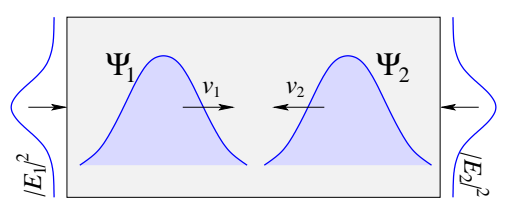

FIG. 1: (a) Level scheme of atoms interacting with weak (quantum) fields $E_{1,2}$ on the transitions $|g\rangle \rightarrow\left|e_{1,2}\right\rangle$ and strong driving fields of Rabi frequencies $\Omega_{1,2}$ on the transitions $\left|e_{1,2}\right\rangle \rightarrow\left|d_{1,2}\right\rangle$, respectively. $V_{\mathrm{dd}}$ denotes the dipoledipole interaction between pairs of atoms in Rydberg states $|d\rangle$. (b) Upon entering the medium, each field having Gaussian transverse intensity profile is converted into the corresponding polariton $\Psi_{1,2}$ representing a coupled excitation of the field and atomic coherence. These polaritons propagate in the opposite directions with slow group velocities $v_{1,2}$ and interact via the dipole-dipole interaction.

respectively. The intermediate states $\left|e_{1,2}\right\rangle$ are resonantly coupled by two strong (classical) driving fields with Rabi frequencies $\Omega_{1,2}$ to the highly excited Rydberg states $\left|d_{1,2}\right\rangle$. In a static electric field $E_{\mathrm{st}} \mathbf{e}_{z}$, the Rydberg states $|d\rangle$ possess large permanent dipole moments $\mathbf{p}=\frac{3}{2} n q e a_{0} \mathbf{e}_{z}$, where $n$ and $q \equiv n_{1}-n_{2}$ are, respectively, the (effective) principal and parabolic quantum numbers, $e$ is the electron charge, and $a_{0}$ is the Bohr radius [17]. A pair of atoms $i$ and $j$ at positions $\mathbf{r}_{i}$ and $\mathbf{r}_{j}$ excited to states $|d\rangle$ interact with each other via the dipole-dipole potential

$$
V_{\mathrm{dd}}=\frac{\mathbf{p}_{i} \cdot \mathbf{p}_{j}-3\left(\mathbf{p}_{i} \cdot \mathbf{e}_{i j}\right)\left(\mathbf{p}_{j} \cdot \mathbf{e}_{i j}\right)}{4 \pi \epsilon_{0}\left|\mathbf{r}_{i}-\mathbf{r}_{j}\right|^{3}},
$$

where $\mathbf{e}_{i j}$ is a unit vector along the interatomic direction. This dipole-dipole interaction results in an energy shift of the pair of Rydberg atoms, while we assume that the state mixing within the same $n$ manifold is suppressed by the proper choice of parabolic $q$ and magnetic $m$ quantum numbers [17, 18]. In the frame rotating with the frequencies of the optical fields, the interaction Hamiltonian has the following form

$$
H=V_{\mathrm{af}}+V_{\mathrm{dd}}
$$

where the atom-field and dipole-dipole interaction terms are given, respectively, by

$$
\begin{aligned}
V_{\mathrm{af}}=-\hbar \sum_{j}^{N}\left[g_{1}^{j} \hat{\mathcal{E}}_{1} \hat{\sigma}_{e_{1} g}^{j}+\Omega_{1} \hat{\sigma}_{d_{1} e_{1}}^{j}\right. \\
\left.\quad+g_{2}^{j} \hat{\mathcal{E}}_{2} \hat{\sigma}_{e_{2} g}^{j}+\Omega_{2} \hat{\sigma}_{d_{2} e_{2}}^{j}+\text { H.c. }\right] \\
V_{\mathrm{dd}}=\hbar \sum_{i>j}^{N} \hat{\sigma}_{d d}^{i} \Delta\left(\mathbf{r}_{i}-\mathbf{r}_{j}\right) \hat{\sigma}_{d d}^{j} .
\end{aligned}
$$

Here $N=\rho V$ is the total number of atoms, $\rho$ being the (uniform) atomic density and $V$ the volume; $\hat{\sigma}_{\mu \nu}^{j} \equiv$ $|\mu\rangle_{j j}\langle\nu|$ is the transition operator of the $j$ th atom; $\hat{\mathcal{E}}_{l}$ is the slowly-varying operator, corresponding to the electric field $E_{l}(l=1,2)$, which obeys the commutation relations $\left[\hat{\mathcal{E}}_{l}(\mathbf{r}), \hat{\mathcal{E}}_{l^{\prime}}^{\dagger}\left(\mathbf{r}^{\prime}\right)\right]=V \delta_{l l^{\prime}} \delta\left(\mathbf{r}-\mathbf{r}^{\prime}\right) ; g_{l}^{j}$ is the corresponding atom-field coupling constant on the transition $|g\rangle_{j} \rightarrow$ $\left|e_{l}\right\rangle_{j}$; and $\hbar \Delta\left(\mathbf{r}_{i}-\mathbf{r}_{j}\right) \equiv{ }_{i}\left\langle\left. d\right|_{j}\left\langle d\left|V_{\mathrm{dd}}\right| d\right\rangle_{i} \mid d\right\rangle_{j}$ is the dipoledipole energy shift for a pair of atoms $i$ and $j$, given by

$$
\Delta\left(\mathbf{r}_{i}-\mathbf{r}_{j}\right)=C \frac{1-3 \cos ^{2} \vartheta}{\left|\mathbf{r}_{i}-\mathbf{r}_{j}\right|^{3}}
$$

where $\vartheta$ is the angle between vectors $\mathbf{e}_{z}$ and $\mathbf{e}_{i j}$, and $C=$ $\wp_{d_{l}} \wp_{d^{\prime}} /\left(4 \pi \epsilon_{0} \hbar\right)$ is a constant proportional to the product of atomic dipole moments $\wp_{d_{l}}=\left\langle d_{l}|\mathbf{p}| d_{l}\right\rangle$ assumed the same for both states $\left|d_{1,2}\right\rangle, \wp_{d_{1,2}}=\wp_{d}$.

Let us introduce collective atomic operators $\hat{\sigma}_{\mu \nu}(\mathbf{r})=$ $\frac{1}{N_{r}} \sum_{j=1}^{N_{r}} \hat{\sigma}_{\mu \nu}^{j}$ averaged over the volume element $d^{3} r$ containing $N_{r}=\rho d^{3} r \gg 1$ atoms around position $\mathbf{r}$. Then Eqs. (2) can be cast in the continuous form

$$
\begin{aligned}
V_{\mathrm{af}} & =-\hbar \rho \int d^{3} r \sum_{l=1,2}\left[g_{l} \hat{\mathcal{E}}_{l} \hat{\sigma}_{e_{l} g}(\mathbf{r})+\Omega_{l} \hat{\sigma}_{e_{l} d_{l}}(\mathbf{r})\right]+\text { H.c. }( \\
V_{\mathrm{dd}} & =\hbar \rho^{2} \iint d^{3} r d^{3} r^{\prime} \hat{\sigma}_{d d}(\mathbf{r}) \Delta\left(\mathbf{r}-\mathbf{r}^{\prime}\right) \hat{\sigma}_{d d}\left(\mathbf{r}^{\prime}\right) .
\end{aligned}
$$

Using Eqs. (3), one can derive a set of HeisenbergLangevin equations for the atomic operators $\hat{\sigma}_{\mu \nu}$ [7]. When the number of photons in the quantum fields $\hat{\mathcal{E}}_{l}$ is much smaller than the number of atoms, these equations can be solved perturbatively in the small parameters $g_{l} \hat{\mathcal{E}}_{l} / \Omega_{l}$ and in the adiabatic approximation for all the fields [16], with the result

$$
\begin{aligned}
\hat{\sigma}_{g e_{l}}(\mathbf{r}) & =-\frac{i}{\Omega_{l}}\left[\frac{\partial}{\partial t}+i \hat{\alpha}(\mathbf{r})\right] \hat{\sigma}_{g d_{l}}(\mathbf{r}) \\
\hat{\alpha}(\mathbf{r}) & =\rho \int d^{3} r^{\prime} \Delta\left(\mathbf{r}-\mathbf{r}^{\prime}\right)\left[\hat{\sigma}_{d_{1} d_{1}}\left(\mathbf{r}^{\prime}\right)+\hat{\sigma}_{d_{2} d_{2}}\left(\mathbf{r}^{\prime}\right)\right] \\
\hat{\sigma}_{g d_{l}}(\mathbf{r}) & =-\frac{g_{l} \hat{\mathcal{E}}_{l}}{\Omega_{l}^{*}}, \quad \hat{\sigma}_{d_{l} d_{l}}(\mathbf{r})=\hat{\sigma}_{d_{l} g}(\mathbf{r}) \hat{\sigma}_{g d_{l}}(\mathbf{r})
\end{aligned}
$$

Let us assume that the transverse profile of both quantum fields is described by a Gaussian $e^{-r_{\perp}^{2} / w^{2}}$ of width $w$, where $r_{\perp}=\left|\mathbf{r}_{\perp}\right|$ is the distance from the field propagation axis, while the Rabi frequencies of classical driving fields $\Omega_{l}$ are uniform over the entire volume $V$. We may then write $g_{l} \hat{\mathcal{E}}_{l}=g_{l}\left(\mathbf{r}_{\perp}\right) \hat{\mathcal{E}}_{l}(z)$, where the traveling-wave electric field operators $\hat{\mathcal{E}}_{l}(z)=\sum_{k} a_{l}^{k} e^{i k z}$ are expressed through the superposition of bosonic operators $a_{l}^{k}$ for the longitudinal field modes $k$, while the (transverseposition-dependent) coupling constants are given by $g_{l}\left(\mathbf{r}_{\perp}\right)=\tilde{g}_{l} e^{-r_{\perp}^{2} / 2 w^{2}}$, with $\tilde{g}_{l}=\left(\wp_{g e_{l}} / \hbar\right) \sqrt{\hbar \omega / 2 \epsilon_{0} V}$, $\wp_{g e_{l}}$ being the dipole matrix element on the transition $|g\rangle \rightarrow\left|e_{l}\right\rangle, V=\pi w^{2} L$, and $L$ the medium length. Under this approximation, the propagation equations for the slowly-varying quantum fields have the form

$$
\left(\frac{\partial}{\partial t} \pm c \frac{\partial}{\partial z}\right) \hat{\mathcal{E}}_{l}(z, t)=i \tilde{g}_{l} N \hat{\sigma}_{g e_{l}}(z),
$$


the sign "+" or "-" corresponding to $l=1$ or 2 , respectively.

Following [16], we introduce new quantum fields $\hat{\Psi}_{l}$ dark state polaritons - via the canonical transformations

$$
\hat{\Psi}_{l}=\cos \theta_{l} \hat{\mathcal{E}}_{l}-\sin \theta_{l} \sqrt{N} \hat{\sigma}_{g d_{l}}
$$

where the mixing angles $\theta_{l}$ are defined through $\tan ^{2} \theta_{l}=$ $\tilde{g}_{l}^{2} N /\left|\Omega_{l}\right|^{2}$. These polaritons correspond to coherent superpositions of electric field $\hat{\mathcal{E}}_{l}$ and atomic coherence $\hat{\sigma}_{g d_{l}}$ operators. Employing the plane-wave decomposition of the polariton operators, one can show that in the weakfield limit, they obey the bosonic commutation relations $\left[\hat{\Psi}_{l}(z), \hat{\Psi}_{l^{\prime}}^{\dagger}\left(z^{\prime}\right)\right] \simeq L \delta_{l l^{\prime}} \delta\left(z-z^{\prime}\right)$. Using Eqs. (4) and (15), we obtain the following propagation equations for the polariton operators,

$$
\left(\frac{\partial}{\partial t} \pm v_{l} \frac{\partial}{\partial z}\right) \hat{\Psi}_{l}(z, t)=-i \sin ^{2} \theta_{l} \hat{\alpha}(z, t) \hat{\Psi}_{l}(z, t) .
$$

Here $v_{l}=c \cos ^{2} \theta_{l}$ is the group velocity, while operator $\hat{\alpha}(z, t)$ is responsible for the self- and cross-phase modulation between the polaritons. It is related to the polariton intensity (excitation number) operators $\hat{\mathcal{I}}_{l} \equiv \hat{\Psi}_{l}^{\dagger} \hat{\Psi}_{l}$ via

$\hat{\alpha}(z, t)=\frac{1}{L} \int_{0}^{L} d z^{\prime} \Delta\left(z-z^{\prime}\right)\left[\sin ^{2} \theta_{1} \hat{\mathcal{I}}_{1}\left(z^{\prime}, t\right)+\sin ^{2} \theta_{2} \hat{\mathcal{I}}_{2}\left(z^{\prime}, t\right)\right]$,

where the 1D dipole-dipole interaction potential $\Delta(z-$ $\left.z^{\prime}\right)$ is obtained after the integration over the transverse profile of the quantum fields,

$$
\begin{aligned}
& \Delta\left(z-z^{\prime}\right)=\frac{1}{\pi w^{2}} \int_{0}^{2 \pi} d \varphi^{\prime} \int_{0}^{\infty} d r_{\perp}^{\prime} r_{\perp}^{\prime} e^{-r_{\perp}^{\prime 2} / w^{2}} \Delta\left(z \mathbf{e}_{z}-\mathbf{r}^{\prime}\right) \\
& =\frac{2 C}{w^{3}}\left[\frac{2\left|z-z^{\prime}\right|}{w}-\sqrt{\pi}\left(1+2 \frac{\left|z-z^{\prime}\right|^{2}}{w^{2}}\right)\right. \\
& \left.\times \exp \left(\frac{\left|z-z^{\prime}\right|^{2}}{w^{2}}\right) \operatorname{erfc}\left(\frac{\left|z-z^{\prime}\right|}{w}\right)\right],
\end{aligned}
$$

and is shown in Fig. 2(a).

It follows from Eq. (7) that the intensity operators $\hat{\mathcal{I}}_{l}$ are constants of motion: $\hat{\mathcal{I}}_{l}(z, t)=\hat{\mathcal{I}}_{l}\left(z \mp v_{l} t, 0\right)$, the upper (lower) sign corresponding to $l=1(l=2)$. Then the formal solution for the polariton operators can be written as

$$
\begin{aligned}
\hat{\Psi}_{l}(z, t)= & \exp \left[-i \sin ^{2} \theta_{l} \int_{0}^{t} d t^{\prime} \hat{\alpha}\left(z \mp v_{l}\left(t-t^{\prime}\right), t^{\prime}\right)\right] \\
& \times \hat{\Psi}_{l}\left(z \mp v_{l} t, 0\right) .
\end{aligned}
$$

Equation (10) is our central result. Let us outline the approximations involved in the derivation of this solution. In order to accommodate the pulses in the medium with negligible losses, their duration $T$ should exceed the inverse of the EIT bandwidth $\delta \omega=$
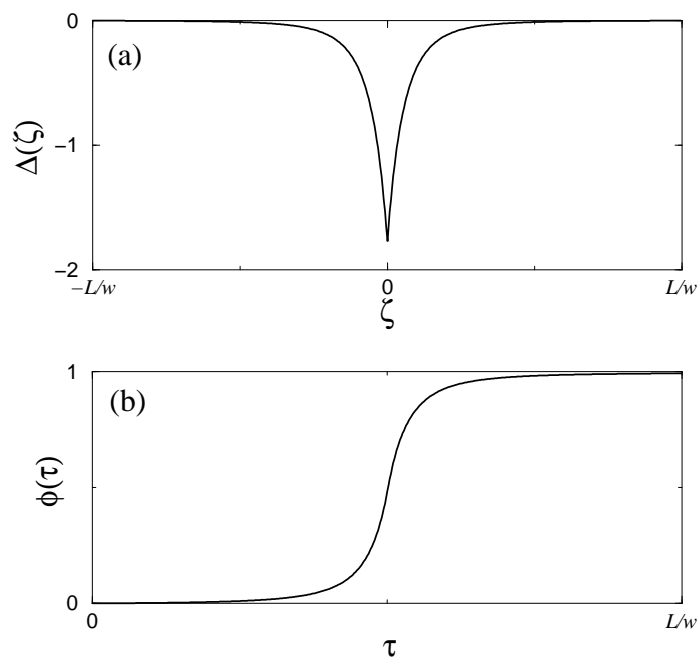

FIG. 2: (a) The 1D dipole-dipole potential $\Delta(\zeta)$ of Eq. 91 as a function of dimensionless distance $\zeta=\left(z-z^{\prime}\right) / w$, in units of $2 C / w^{3} \mathrm{~Hz}$. (b) The resulting phase-shift $\phi(\tau) \equiv$ $\phi(v t, L-v t, t)$ of Eq. (14) as a function of dimensionless time $\tau=v t / w$, in units of $2 C /\left(v w^{2}\right)$ rad.

$\left|\Omega_{l}\right|^{2}\left(\gamma_{g e_{l}} \sqrt{\kappa_{0} L}\right)^{-1}$, where $\gamma_{g e_{l}}$ is the transversal relaxation rate and $\kappa_{0} \simeq 3 \lambda^{2} /(2 \pi) \rho$ is the resonant absorption coefficient on the transition $|g\rangle \rightarrow\left|e_{l}\right\rangle$. This yields the condition $\left(\kappa_{0} L\right)^{-1 / 2} \ll T v_{l} / L<1$ which requires a medium with large optical depth $\kappa_{0} L \gg 1$ [16. In addition, the dipole-dipole energy shift should lie within the EIT bandwidth $\delta \omega$ for all $\left|z-z^{\prime}\right| \leq L$, which implies that $|\Delta(0)|=2 \sqrt{\pi} C / w^{3}<\delta \omega$. Finally, the propagation/interaction time of the two pulses $t_{\text {out }}=L / v_{l}$ is limited by the relaxation rate of the Rydberg states $\gamma_{d_{l}}$ via $t_{\text {out }} \gamma_{d_{l}} \ll 1$.

From now on, we assume that $\theta_{1,2}=\theta$, i.e., $\tilde{g}_{1}^{2} N /\left|\Omega_{1}\right|^{2}=\tilde{g}_{2}^{2} N /\left|\Omega_{2}\right|^{2}$, which yields $v_{1,2}=v=c \cos ^{2} \theta$. We are interested in the evolution of input state

$$
\left|\Phi_{\text {in }}\right\rangle=\left|1_{1}\right\rangle \otimes\left|1_{2}\right\rangle,
$$

composed of two single-excitation polariton wavepackets

$$
\left|1_{l}\right\rangle=\frac{1}{L} \int d z f_{l}(z) \hat{\Psi}_{l}(z)^{\dagger}|0\rangle
$$

where $f_{l}(z)$ define the spatial envelopes of the corresponding wavepackets $l=1,2$ which initially (at $t=0$ ) are localized around $z=0, L$, respectively. For such an initial state, all the relevant information is contained in the expectation values of the polariton intensities $\left\langle\hat{\mathcal{I}}_{l}(z, t)\right\rangle=\left\langle\Phi_{\text {in }}\left|\hat{\mathcal{I}}_{l}(z, t)\right| \Phi_{\text {in }}\right\rangle$ and the two-particle wavefunction [7, 11, 12]

$$
F_{12}\left(z_{1}, z_{2}, t\right)=\left\langle 0\left|\hat{\Psi}_{1}\left(z_{1}, t\right) \hat{\Psi}_{2}\left(z_{2}, t\right)\right| \Phi_{\text {in }}\right\rangle .
$$

With the above solution, for the polariton intensities we have $\left\langle\hat{\mathcal{I}}_{1,2}(z, t)\right\rangle=\left\langle\hat{\mathcal{I}}_{1,2}(z \mp v t, 0)\right\rangle=\left|f_{1,2}(z \mp v t)\right|^{2}$, which describes the shape-preserving counter-propagation of 
the two polaritons with group velocity $v$. Substituting the operator solution (10) into (12), after some algebra, we obtain the following expression for the two-particle wavefunction

$$
\begin{aligned}
F_{12}\left(z_{1}, z_{2}, t\right) & =f_{1}\left(z_{1}-v t\right) f_{2}\left(z_{2}+v t\right) \exp \left[i \phi\left(z_{1}, z_{2}, t\right)\right], \\
\phi\left(z_{1}, z_{2}, t\right) & =-\sin ^{4} \theta \int_{0}^{t} d t^{\prime} \Delta\left(z_{1}-z_{2}-2 v\left(t-t^{\prime}\right)\right),
\end{aligned}
$$

which indicates that the dipole-dipole interaction between the two single-excitation polaritons results in the conditional phase-shift $\phi\left(z_{1}, z_{2}, t\right)$. We consider a situation in which at time $t=0$, the first pulse is localized at $z_{1}=0$ and the second pulse is at $z_{2}=L$, while after the interaction, at time $t_{\text {out }}=L / v$, the coordinates of the two pulses are $z_{1}=L$ and $z_{2}=0$, respectively [Fig. 2(b)]. Then the phase-shift accumulated during the interaction is spatially uniform, and is given by

$$
\phi(L, 0, L / v)=-\frac{\sin ^{4} \theta}{v} \int_{0}^{L} d z^{\prime} \Delta\left(2 z^{\prime}-L\right)=\frac{2 C \sin ^{4} \theta}{v w^{2}} .
$$

This remarkably simple result is obtained upon replacing the variable $\left(2 z^{\prime}-L\right) / w \rightarrow \zeta^{\prime}$ and extending the integration limits to $L / w \rightarrow \infty$. The main limitation on the phase shift is imposed by the condition $|\Delta(0)|<\delta \omega$. In terms of experimentally relevant parameters, the group velocity is $v \simeq 2|\Omega|^{2} /\left(\kappa_{0} \gamma_{g e}\right) \ll c\left(\sin ^{2} \theta \simeq 1\right)$, and we have $\phi<\frac{1}{2} w \sqrt{\kappa_{0} / \pi L}$.

To relate the foregoing discussion to a realistic experiment, let us assume an ensemble of cold alkali atoms in the ground state $|g\rangle$ with density $\rho \sim 10^{14} \mathrm{~cm}^{-3}$ confined in a trap of length $L \sim 100 \mu \mathrm{m}$. The resonant quantum fields with $\lambda \sim 0.5 \mu \mathrm{m}$ have the transverse width $w \sim 30 \mu \mathrm{m}$. In the presence of driving fields with appropriate frequencies, the single-photon pulses lead to the (two-photon) excitation of single atoms to the Rydberg states $|d\rangle$ with quantum numbers $n \simeq 25$ and $q=n-1$. The corresponding dipole moments are $\wp_{d} \simeq 900 e a_{0}$, while $\gamma_{d} \sim 2 \times 10^{3} \mathrm{~s}^{-1}$ 17. With $\gamma_{g e} \sim 10^{7} \mathrm{~s}^{-1}$ and $\Omega \sim 1.6 \times 10^{7} \mathrm{rad} / \mathrm{s}$, the group velocity is $v \simeq 4 \mathrm{~m} / \mathrm{s}$, and the accumulated phase shift is $\phi \simeq \pi$ with the fidelity $F=\exp \left(-\gamma_{d} L / v\right) \gtrsim 0.95$.

To summarize, we have studied a novel highly-efficient scheme for cross-phase modulation and entanglement of two counterpropagating single-photon wavepackets, employing their ultra-small group velocities in atomic vapors, under the conditions of electromagnetically induced transparency, and the strong long-range dipole-dipole interactions of the accompanying Rydberg-state excitations in a ladder-type field-atom coupling setup. We have solved, in the weak-field and adiabatic approximations, the effective one-dimensional propagation equations for the polariton operators and have shown that the dipole- dipole interaction leads to a homogeneous conditional phase shift that reach the value of $\pi$ even if the transverse cross section of the pulses $w^{2}$ is much (three orders of magnitude) larger than the diffraction limit $\lambda^{2}$. This is the obvious merit of the present proposal, as compared to previous schemes based on local interactions of photons or slow-light polaritons 9, 10, 11, 12, 13, 14, 15], which require the photonic beam cross section to be comparable to the cross section for atomic resonant absorption. Hence, our proposal paves the way to the coveted deterministic entanglement of two single-photon pulses and the realization of the universal photonic phase gate [13].

This work was supported by the EC (QUACS RTN and ATESIT network), ISF, and Minerva.

[1] M. Nielsen and I. Chuang, Quantum Computation and Quantum Information, (Cambridge University Press, Cambridge, 2000).

[2] D.P. DiVincenzo, Fortschr. Phys. 48, 711 (2000).

[3] I.L. Chuang and Y. Yamamoto, Phys. Rev. A 52, 3489 (1995).

[4] E. Knill, R. Laflamme, and G.J. Milburn, Nature 409, 46 (2001); J.L. O'Brien et al., Nature 426, 264 (2003); S. Gasparoni et al., Phys. Rev. Lett. 93, 020504 (2004).

[5] Q. A. Turchette et al., Phys. Rev. Lett. 75, 4710 (1995); A. Imamoglu, H. Schmidt, G. Woods, and M. Deutsch, Phys. Rev. Lett. 79, 1467 (1997); A. Rauschenbeutel et al., Phys. Rev. Lett. 83, 5166 (1999).

[6] S. E. Harris, Phys. Today 50(7), 36 (1997); M. D. Lukin, Rev. Mod. Phys. 75, 457 (2003).

[7] M.O. Scully and M.S. Zubairy, Quantum Optics (Cambridge University Press, Cambridge, UK, 1997).

[8] L.V. Hau, S.E. Harris, Z. Dutton, and C.H. Behroozi, Nature 397, 594 (1999); M.M. Kash et al., Phys. Rev. Lett. 82, 5229 (1999); D. Budker, D.F. Kimball, S.M. Rochester, and V.V. Yashchuk, Phys. Rev. Lett. 83, 1767 (1999).

[9] H. Schmidt and A. Imamoğlu, Opt. Lett. 21, 1936 (1996).

[10] S. Harris and L. Hau, Phys. Rev. Lett. 82, 4611 (1999).

[11] M.D. Lukin and A. Imamoğlu, Phys. Rev. Lett. 84, 1419 (2000).

[12] D. Petrosyan and Yu. P. Malakyan, Phys. Rev. A 70, 023822 (2004).

[13] I. Friedler, G. Kurizki and D. Petrosyan, Europhys. Lett. 68, 625 (2004); Phys. Rev. A 71, 023803 (2005).

[14] A. Andre M. Bajcsy, A.S. Zibrov, and M.D. Lukin, Phys. Rev. Lett. 94, 063902 (2005).

[15] M. Masalas and M. Fleischhauer, Phys. Rev. A 69, 061801(R) (2004).

[16] M. Fleischhauer and M.D. Lukin, Phys. Rev. Lett. 84, 5094 (2000); Phys. Rev. A 65, 022314 (2002).

[17] T.F. Gallagher, Rydberg Atoms (Cambridge University Press, Cambridge, 1994).

[18] D. Jaksch et al., Phys. Rev. Lett. 85, 2208 (2000). 